\title{
Rare murmur in a patient with constrictive pericarditis
}

\author{
Yukio Abe $\odot$, Toshio Saito, Takahiko Naruko \\ Department of Cardiology, Osaka City General Hospital, Osaka, Japan
}

An 80-year-old man was admitted with severe limb edema. A harsh sound at early diastole was audible by auscultation at the left sternal border. The computed tomography from a referral clinic revealed a thick pericardial layer with a severely calcified surface around the right ventricular free wall (Fig. 1A). He was diagnosed by echocardiography with right-sided heart failure due to constrictive pericarditis $(\mathrm{CP})$ and mild to moderate pulmonary regurgitation (PR) at early diastole (Fig. 1B). A phonocardiogram showed a high-pitched regurgitant murmur at the left sternal border with a short duration during early diastole right after the pulmonary component of the second heart sound (Fig. 1C). The PR arising from the gradient with rapid deceleration between pulmonary artery pressure and the CP-induced dip of the right ventricular pressure was proven by right heart catheterization as the cause of the rare murmur (Fig. 1D, Suppl. Video with sound). He had a history of pulmonary tuberculosis. Consequently, tuberculous pericarditis was thought to be the cause of his $\mathrm{CP}$, and a surgical pericardial dissection was performed. The intensity of the diastolic murmur became smaller, and the duration became longer after the surgery. Early diastolic PR with rapid deceleration of the gradient between the pulmonary artery pressure and the dip of the right ventricular pressure in patients with $\mathrm{CP}$ has been previously reported using Doppler echocardiography, whereas the corresponding murmur has not. According to available research, this is the first report of the dip-induced harsh PR murmur during early diastole in a patient with constrictive pericarditis.

Conflict of interest: None declared

Address for correspondence: Yukio Abe, MD, PhD, Department of Cardiology, Osaka City General Hospital, 2-13-22 Miyakojima-hondori, Miyakojima-ku, Osaka 534-0021, Japan, tel: +81-6-6929-1221, fax: +81-6-6929-1090, e-mail: abeyukio@aol.com

Received: 5.12.2020 Accepted: 28.02.2020

This article is available in open access under Creative Common Attribution-Non-Commercial-No Derivatives 4.0 International (CC BY-NC-ND 4.0) license, allowing to download articles and share them with others as long as they credit the authors and the publisher, but without permission to change them in any way or use them commercially. 


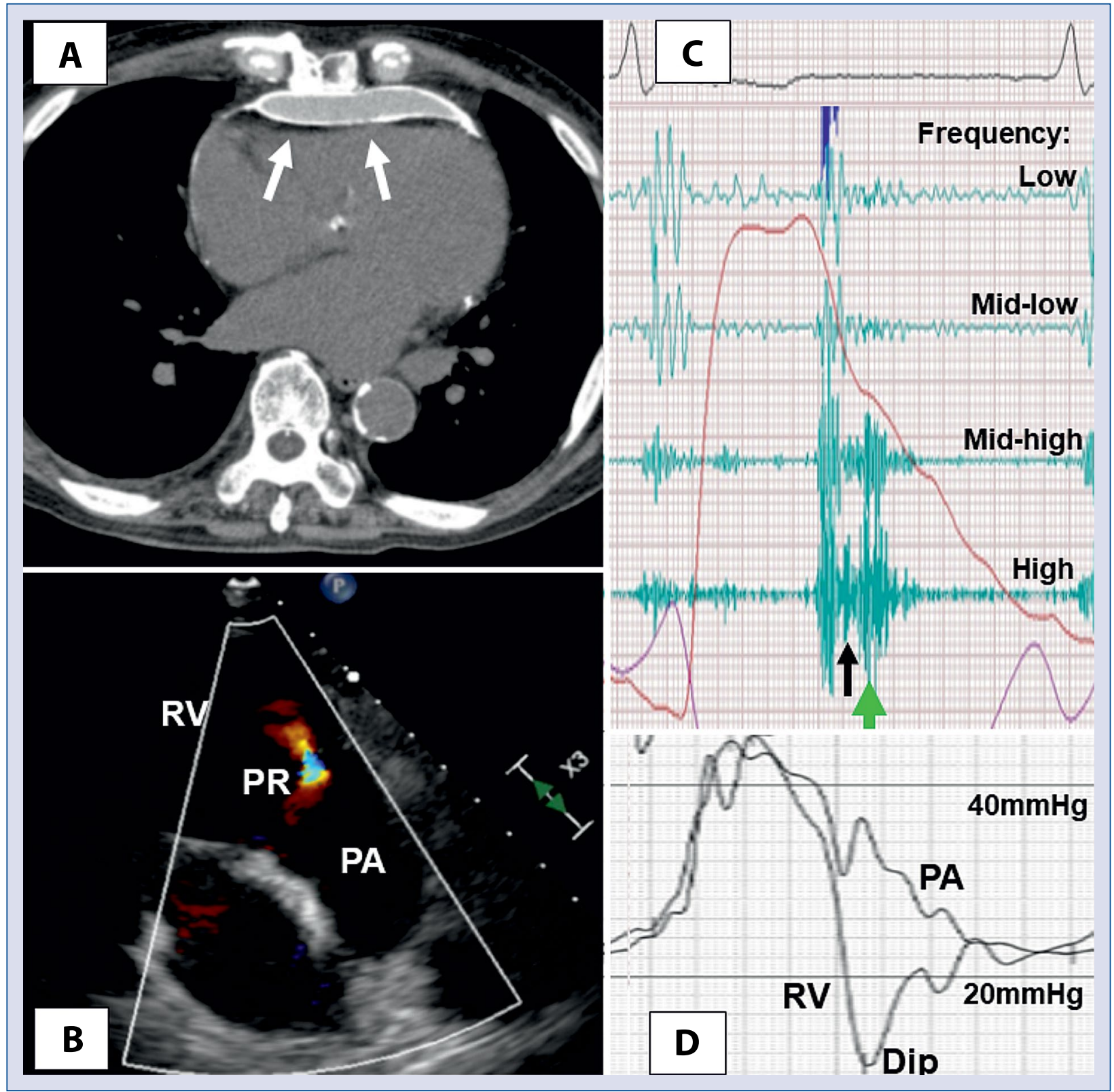

Figure 1. A. A calcified pericardial layer in the computed tomography (white arrows); B. Moderate pulmonary regurgitation (PR) in echocardiography; C. A high-pitched murmur (green arrow) during early diastole right after the pulmonary component (black arrow) of the second heart sound in a phonocardiogram recorded at the left sternal border; D. The gradient with rapid deceleration between pulmonary artery (PA) pressure and the dip of the right ventricular (RV) pressure recorded at catheterization. 
